\title{
Ceramide: a contributor to insulin resistance or an innocent bystander?
}

\author{
G. Boden
}

Published online: 6 May 2008

(C) Springer-Verlag 2008

Keywords Ceramide - Diacylglycerol .

Inflammatory cytokines $\cdot$ Insulin receptor substrate $1 / 2$. Insulin resistance $\cdot$ Liposyn II · Obesity · Protein kinase C . Protein kinase B/Akt $\cdot$ Skeletal muscle

Many studies in humans and animals have clearly demonstrated that obesity predisposes to the development of insulin resistance. Despite years of investigational efforts, however, it is still not completely known why and how this happens. One currently popular hypothesis is that obesity triggers a low-grade, chronic inflammation and the release of proinflammatory cytokines from adipose tissue and macrophages, which can antagonise insulin action [1]. According to another hypothesis, chronic excessive nutrient intake results in accumulation of fat not only in the physiological storage sites, i.e. in adipose tissue, but also in other tissues such as liver and skeletal muscle, where it causes problems [2]. This model is supported by studies demonstrating that experimental manipulations promoting lipid deposition in muscle and/or liver such as i.v. lipid/heparin infusions or overproduction of lipoprotein lipase in muscle and/or liver of transgenic mice, cause insulin resistance [3]. It has been shown, however, that intramyocellular or intrahepatic accumulation of triacylglycerol is not directly responsible for decreased insulin action, but is likely to be the source for accumulation of several lipid metabolites that can inhibit insulin signalling. For instance, experiments in animals and humans have shown that short-term lipid/ heparin infusions not only increase intramyocellular triacylglycerol concentrations, but also result in intracellular

\section{G. Boden $(\bowtie)$}

Division of Endocrinology, Diabetes and Metabolism,

Temple University Hospital,

3401 North Broad Street,

Philadelphia, PA 19140, USA

e-mail: bodengh@tuhs.temple.edu accumulation of long-chain acyl-CoA and diacylglycerol and activation of several serine/threonine kinases, including several protein kinase $\mathrm{C}$ isoforms, inhibitor of kappa-B kinase and c-jun N-terminal kinase [4]. Some of these kinases have been shown to interfere with insulin signalling by decreasing tyrosine phosphorylation of IRS-1/2. Diacylglycerol, rather than long-chain acyl-CoA, has been implicated as the most likely lipid metabolite responsible for the lipid-induced insulin resistance [5] (Fig. 1).

The ceramides, a family of molecules consisting of variable length fatty acids linked to sphingosine or related bases, are another type of lipid metabolite postulated to be a link between nutrient excess, inflammatory cytokine production and development of insulin resistance (Fig. 1). Supporting this notion are many in vitro and animal studies showing that ceramide can induce insulin resistance [6]. One of the mechanisms by which ceramide can inhibit insulin action in isolated cells involves inhibition of Akt. This can occur through several mechanisms, including dephosphorylation of Akt by increased protein phosphatase 2 activity, or by inhibition of translocation of Akt to the cell membrane [6].

Whether ceramide is involved in the development of obesity-related insulin resistance in humans is less clear. The paper by Skovbro et al. [7] in this issue of Diabetologia addresses this question. Ceramide content was measured in muscle biopsy samples taken from 33 men with a wide range of insulin sensitivities (determined by euglycaemichyperinsulinaemic clamp), ranging from very low (patients with type 2 diabetes) to very high (endurance-trained healthy men). The authors found that basal muscle ceramide concentration was similar in all men, regardless of the large differences in insulin sensitivity, and did not change in response to hyperinsulinaemia. They concluded that muscle ceramide content could not be a major factor in the pathogenesis of the insulin resistance in these men. Their results, however, are in disagreement with human studies by others. For instance, Straczkowski et al. [8] 


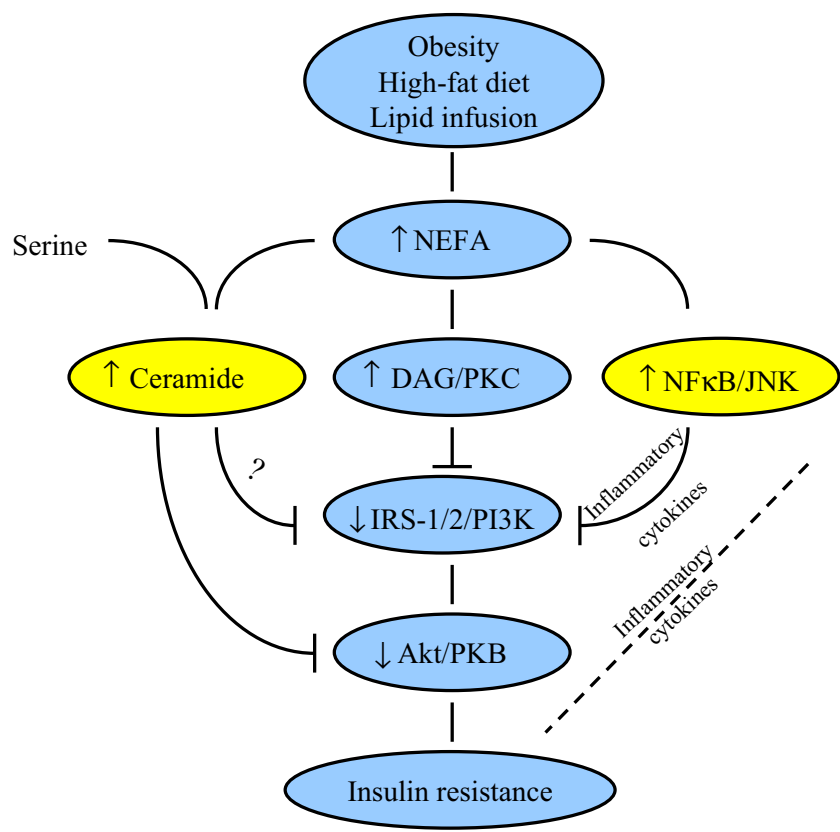

Fig. 1 Model for putative effects of ceramide on insulin signalling. Ceramide inhibits Akt activation by dephosphorylation and inhibition of translocation (see text). This may result in insulin resistance. Whether ceramide also inhibits tyrosine phosphorylation of IRS-1/2 and activation of PI3K remains controversial. The dotted line indicates that the relationship is not established. Different colours (yellow and blue) indicate different pathways through which NEFA may affect insulin action. DAG, diacylglycerol; IKK, inhibitor of $\mathrm{KB}$ kinase; JNK, c-jun $\mathrm{N}$ terminal kinase; PI3K, phosphatidylinositol 3-kinase; $\mathrm{PKC}$, protein kinase $\mathrm{C}$

reported a threefold higher total ceramide content in muscle biopsy samples from obese men with impaired glucose tolerance compared with lean men with normal glucose tolerance. Similarly, Adams et al. [9] evaluated ceramide content in muscle biopsy samples obtained from lean insulin-sensitive and obese insulin-resistant men and found that muscle ceramide content was around two times higher in the obese compared with the lean subjects.

It is difficult to reconcile these different results because the study populations (exclusively men) were comparable (albeit the numbers were relatively small), and the biopsy samples were obtained from the same site (vastus lateralis muscles). It is, of course possible that the results could have been skewed because of unidentified factors, such as prestudy differences in food intake or physical activity. However, total muscle ceramide content in obese patients with type 2 diabetes in the study by Skovbro et al. [7] was approximately six times lower than that in obese patients with impaired glucose tolerance in the study by Straczkowski et al. ( $\sim 150 \mathrm{vs} \sim 900 \mathrm{nmol} / \mathrm{g}$ tissue) [8], which suggests that the different results may have been due, at least in part, to methodological problems. Moreover, ceramide, because of its highly hydrophobic nature, is confined to cell membranes, and there are ceramide rich regions within membranes (called rafts) that constitute important signalling microdomains [10]. Thus, measuring ceramide in total lipid extracts from a small piece of homogenised muscle could theoretically obscure local differences. Also, measuring ceramide content rather than bioactivity may be misleading, as ceramide can be inactivated, for instance by glycosylation [11]. There are examples of lipid-induced insulin resistance, such as that induced by i.v. administration of Liposyn II (Abbott Laboratories, Chicago, IL, USA), which do not involve a change in muscle ceramide content [12]. This, however, can be explained by the fact that the majority $(\sim 88 \%)$ of fatty acids in Liposyn II are unsaturated (C18:1, C18:2, C18:3), and cannot be used for de novo synthesis of ceramide. In conclusion, the question of whether ceramide contributes to obesity-associated insulin resistance in humans, and, if so, to what extent, is unresolved at present and will require additional studies.

Acknowledgements Supported by National Institutes of Health grants R01-DK58895, HL-0733267 and DK-66003.

Duality of interest The author does not have a duality of interest.

\section{References}

1. Wellen KE, Hotamisligil GS (2005) Inflammation, stress and diabetes. J Clin Invest 115:1111-1119

2. Unger RH (2003) Minireview: weapons of lean body mass destruction: the role of ectopic lipids in the metabolic syndrome. Endocrinology 144:5159-5165

3. Boden G, Shulman GI (2002) Free fatty acids in obesity and type 2 diabetes: defining their role in the development of insulin resistance and beta-cell dysfunction. Eur J Clin Invest 32:14-23

4. Boden G (2006) Obesity, insulin resistance, type 2 diabetes and free fatty acids. Expert Rev Endocrinol Metab 1:499-505

5. Yu C, Chen Y, Zong H et al (2002) Mechanisms by which fatty acids inhibit insulin activation of IRS-1 associated phosphatidylinositol 3-kinase activity in muscle. J Biol Chem 277:50230-50236

6. Summers SA (2006) Ceramide in insulin resistance and lipotoxicity. Prog Lipid Res 45:42-72

7. Skovbro M, Baranowski M, Skov-Jensen C et al. (2008) Human muscle ceramide content is not a major factor in muscle insulin sensitivity. Diabetologia. DOI 10.1007/s00125-008-1014-z

8. Straczkowski M, Kowalska I, Baranowski M et al (2007) Increased skeletal muscle ceramide level in men at risk of developing type 2 diabetes. Diabetologia 50:2366-2373

9. Adams JM, Pratipanawatr T, Berria R et al (2004) Ceramide content is increased in skeletal muscle from obese insulin-resistant humans. Diabetes 53:25-31

10. Ruvolo PP (2003) Intracellular signal transduction pathways activated by ceramide and its metabolites. Pharmacol Res 47:383-392

11. Liu YY, Han TY, Giuliano AE, Hansen N, Cabot MC (2000) Uncoupling ceramide glycosylation by transfection of glucosylceramide synthase antisense reverses adriamycin resistance. J Biol Chem 275:7138-7143

12. Itani SI, Ruderman NB, Schmieder F, Boden G (2002) Lipidinduced insulin resistance in human muscle is associated with changes in diacylglycerol, protein kinase C, and IkappaB-alpha. Diabetes 51:2005-2011 